\title{
Suspected Anaphylactic Shock Associated with Rocuronium in an Infant: A Case Report
}

\author{
Hideya Katoh, Yoshifumi Naito, Chihiro Aoki, Mao Kinoshita, Yoshinobu Nakayama, \\ Teiji Sawa* \\ Department of Anesthesiology, Kyoto Prefectural University of Medicine, Kyoto, Japan \\ Email: *anesth@koto.kpu-m.ac.jp
}

Received 3 March 2016; accepted 5 April 2016; published 8 April 2016

Copyright (C) 2016 by authors and Scientific Research Publishing Inc.

This work is licensed under the Creative Commons Attribution International License (CC BY). http://creativecommons.org/licenses/by/4.0/

(c) (i) Open Access

\begin{abstract}
We report a case of severe anaphylactic shock in a 5-month-old infant who was scheduled to undergo an external inguinal hernia repair under general anesthesia. Rocuronium used for anesthesia induction was the most likely cause of anaphylaxis. High levels of serum tryptase and histamine detected in the blood sample collected during the anaphylactic reaction confirmed the diagnosis of anaphylactic shock. The patient's clinical status improved within 90 min of intervention by the intravenous injection of vasopressors and a steroid. Surgery was canceled, and the patient stayed in the pediatric intensive care unit (PICU) under artificial ventilation for $5 \mathrm{~h}$ before safe extubation. The patient achieved full recovery the next day, without any sequelae. The rescheduled surgery was successfully completed 5 months later under general anesthesia without the use of neuromuscular blocking agents.
\end{abstract}

\section{Keywords}

Anaphylaxis, Neuromuscular Blocking Agent, Rocuronium, Shock

\section{Introduction}

The reported incidence of perioperative anaphylaxis ranges between 1:3500 and 1:20,000, with a mortality rate of 3.4\% [1]. Although any medication can potentially cause perioperative anaphylaxis, neuromuscular blocking agents (NMBAs) are the most common cause, with 58.2\% of all cases [2] and an overall incidence of 1:6500 in those receiving NMBAs [3]. In this report, we present a case of suspected anaphylactic shock associated with rocuronium in anesthesia induction of an infant who was planned to undergo external inguinal herniation surgery.

${ }^{*}$ Corresponding author.

How to cite this paper: Katoh, H., Naito, Y., Aoki, C., Kinoshita, M., Nakayama, Y. and Sawa, T. (2016) Suspected Anaphylactic Shock Associated with Rocuronium in an Infant: A Case Report. Open Journal of Anesthesiology, 6, 51-54.

http://dx.doi.org/10.4236/ojanes.2016.64008 


\section{Case Description}

A 5-month-old, $7.1 \mathrm{~kg}$ male infant was scheduled for an external inguinal hernia repair under general anesthesia. Anesthesia was induced using the slow induction method, with the inhalation of a mixture of oxygen, nitrous oxide, and 2.5\% sevoflurane, as well as subsequent intravenous injections of atropine sulfate (0.1 mg) and rocuronium (7.5 mg). Anesthesia was maintained under controlled ventilation using a mixture of air, oxygen, and $2 \%$ sevoflurane, with a small dose of intravenous fentanyl $(5 \mu \mathrm{g})$. Approximately $3 \mathrm{~min}$ after tracheal intubation, erythema, facial edema, tachycardia, and hypotension were observed, and manual ventilation suddenly became very difficult. The patient's transdermal systolic blood pressure decreased to $40 \mathrm{mmHg}$ (Figure 1).

The treatment for anaphylactic shock was immediately initiated with intravenous bolus injections of phenylephrine $(0.01 \mathrm{mg}$ ) given six times, followed by ephedrine $(0.4 \mathrm{mg})$ bolus injections given three times. His blood pressure gradually increased to $100 / 50 \mathrm{mmHg}$. Emergency invasive blood pressure measurement from the radial artery was established, and the scheduled surgery was cancelled. Methylprednisolone (50 mg) was also administered. After approximately1 h of observation in the operating room, the patient was transferred to the pediatric intensive care unit (PICU) under artificial ventilation, with intravenous midazolam for sedation. The patient was administered antihistaminic drugs and sedated with a continuous intravenous infusion of midazolam and dexmedetomidine in the PICU. His vitals became stable during the next $5 \mathrm{~h}$, and he was safely extubated after no evidence of laryngeal edema was found by laryngoscopy. The next morning, he was transferred to the general pediatric ward and was discharged on the fifth day after the anaphylactic shock.

Subsequently, serum tryptase, histamine, and non-specific immunoglobulin (Ig)E levels in the blood sample collected $1 \mathrm{~h}$ after the shock were measured. Serum tryptase and histamine levels were high at $15.8 \mu \mathrm{g} / \mathrm{L}$ (normal, $<8 \mu \mathrm{g} / \mathrm{L})$ and $1.95 \mathrm{ng} / \mathrm{ml}(0.15-1.23 \mathrm{ng} / \mathrm{mL})$, respectively; however, the non-specific IgE level was within normal limits at $102 \mathrm{IU} / \mathrm{mL}$. Two months after discharge, allergen tests performed by the pediatric department

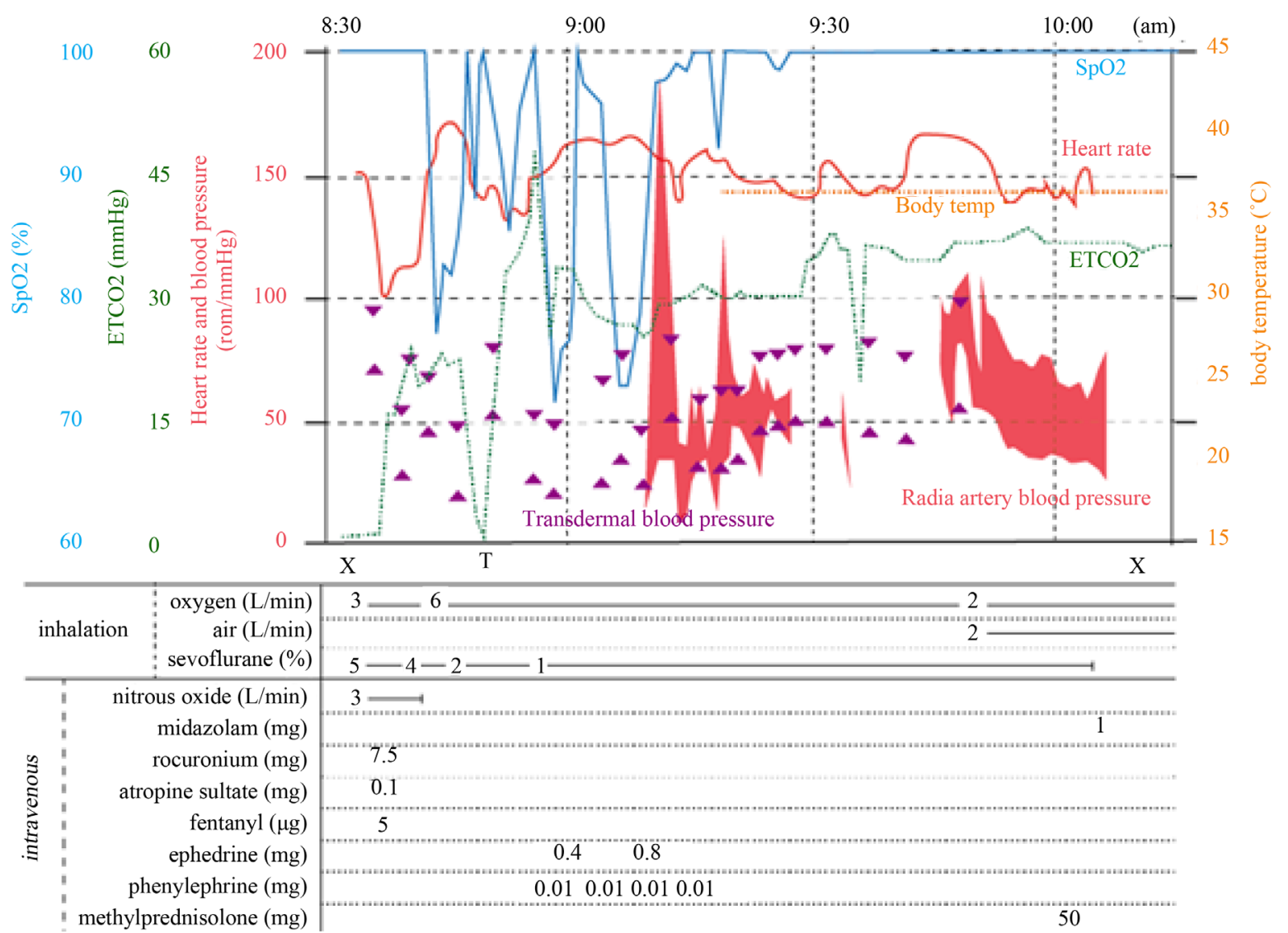

Figure 1. Record of the general anesthesia performed on a 5-month-old infant demonstrating anaphylactic shock during anesthesia induction. X, start and end of anesthesia; $\mathrm{T}$, intubation. 
detected many food sources, including milk, wheat, rice, egg yolk, egg white, and ovomucoid, as allergens in this patient.

Five months after the anaphylactic shock, the external inguinal hernia repair was performed. Before anesthesia, a route for intravenous injections was established at the pediatric ward while the patient was awake. In the operating room, after the intravenous injection of atropine sulfate, general anesthesia was induced with the inhalation of a mixture of nitrous oxide, oxygen, and 5\% sevoflurane, and suxamethonium chloride for laryngospasm was prepared for emergency use. After sufficient anesthesia was achieved with inhalation by mask ventilation, a supraglottic airway device (Laryngeal Mask Proseal ${ }^{\mathrm{TM}}$; size, 1.5 ) was inserted through the oral cavity with no use of muscle relaxants or narcotics. Anesthesia with spontaneous respiration was maintained under the continuous inhalation of a mixture of nitrous oxide, oxygen, and 2.5\% - 3.0\% sevoflurane. Surgery was completed without complications. The patient was extubated in the operating room after recovery from anesthesia with no problems and was discharged the next day.

\section{Discussion}

Several epidemiological studies and case reports about rocuronium and anaphylaxis have been reported up until today [4]-[7]. However, rocuronium-induced anaphylaxis in infants must be very rare because we cannot easily find it in the public databases. Based on the high levels of serum tryptase and histamine, this patient was later confirmed to have experienced an anaphylactic shock. During anaphylaxis, serum tryptase was reported to reach peak levels approximately $1 \mathrm{~h}$ after shock in anaphylaxis cases [8], which was also observed in this patient. Recent studies have demonstrated that rocuronium has a higher risk of inducing IgE-mediated anaphylaxis than other muscle relaxant agents [9] [10]. Radioimmunoassay for IgE antibodies to specific NMBAs proved to be both more sensitive and efficient [11] [12]. Although the serum level of non-specific IgE was within the normal range in this patient, we could not rule out the possibility of IgE-mediated anaphylaxis because NMBA-reactive IgE antibodies were not specifically measured. Intradermal allergen testing of rocuronium was debated in this patient, which was not performed as the test itself could induce anaphylaxis again. Further, intradermal tests were shown to produce false-positive reactions in some cases [13]. Numerous recent reports demonstrated that sugammadex, a selective relaxant binding agent, might be able to improve recovery in cases of anaphylaxis triggered by rocuronium [14] [15]. However, sugammadex was not used in this case because his condition improved after the administration of vasopressors and a steroid as standard treatments we provided for anaphylactic shock.

\section{Conclusion}

In summary, we report a case of severe anaphylactic shock in a 5-month-old infant who was scheduled to undergo external inguinal hernia repair under general anesthesia. Rocuronium used during anesthesia induction was the most likely causative agent. High levels of serum tryptase and histamine supported the diagnosis of anaphylactic shock. The planned surgery was canceled, and the rescheduled surgery was successfully completed 5 months later under general anesthesia with a supraglottic airway device and in the absence of any NMBAs. This case gives us a caution that rocuronium can be a causative of severe anaphylactic shock even in infants.

\section{Acknowledgements}

Authors thank the patient's parent for their written permission to publish the case.

\section{Funding}

None.

\section{Conflict of Interest}

None.

\section{References}

[1] Mertes, P.M. and Volcheck, G.W. (2015) Anaphylaxis to Neuromuscular-Blocking Drugs: All Neuromuscular-Blocking 
Drugs Are Not the Same. Anesthesiology, 122, 5-7. http://dx.doi.org/10.1097/ALN.0000000000000516

[2] Mertes, P.M., Laxenaire, M.C., Alla, F. and Groupe d'Etudes des Reactions Anaphylactoides, P. (2003) Anaphylactic and Anaphylactoid Reactions Occurring during Anesthesia in France in 1999-2000. Anesthesiology, 99, 536-545. http://dx.doi.org/10.1097/00000542-200309000-00007

[3] Mertes, P.M., Laxenaire, M.C., Lienhart, A., Aberer, W., Ring, J., Pichler, W.J., et al. (2005) Reducing the Risk of Anaphylaxis during Anaesthesia: Guidelines for Clinical Practice. Journal of Investigational Allergology and Clinical Immunology, 15, 91-101.

[4] Laxenaire, M.C. and Mertes, P.M. (2001) Anaphylaxis during Anaesthesia. Results of a Two-Year Survey in France. British Journal of Anaesthesia, 87, 549-558. http://dx.doi.org/10.1093/bja/87.4.549

[5] Choi, S.H., Yi, J.W. and Rha, Y.H. (2013) Rocuronium Anaphylaxis in a 3-Year-Old Girl with No Previous Exposure to Neuromuscular Blocking Agents. Asian Pacific Journal of Allergy and Immunology, 1, 163-166. http://dx.doi.org/10.12932/ap0256.31.2.2013

[6] Sadleir, P.H., Clarke, R.C., Bunning, D.L. and Platt, P.R. (2013) Anaphylaxis to Neuromuscular Blocking Drugs: Incidence and Cross-Reactivity in Western Australia from 2002 to 2011. British Journal of Anaesthesia, 110, 981-987. http://dx.doi.org/10.1093/bja/aes506

[7] Jang, Y.H., Kim, S.G., Son, Y.H. and Park, J.M. (2010) Rocuronium Bromide Induced Anaphylaxis in a Child—A Case Report. Korean Journal of Anesthesiology, 59, 411-415. http://dx.doi.org/10.4097/kjae.2010.59.6.411

[8] Harper, N.J., Dixon, T., Dugue, P., Edgar, D.M., Fay, A., Gooi, H.C., et al. (2009) Suspected Anaphylactic Reactions Associated with Anaesthesia. Anaesthesia, 64, 199-211. http://dx.doi.org/10.1111/j.1365-2044.2008.05733.x

[9] Sadleir, P.H., Clarke, R.C., Bunning, D.L. and Platt, P.R. (2013) Anaphylaxis to Neuromuscular Blocking Drugs: Incidence and Cross-Reactivity in Western Australia from 2002 to 2011. British Journal of Anaesthesia, 110, 981-987. http://dx.doi.org/10.1093/bja/aes506

[10] Takazawa, T., Mitsuhata, H. and Mertes, P.M. (2015) Sugammadex and Rocuronium-Induced Anaphylaxis. Journal of Anesthesia, 30, 290-297. http://dx.doi.org/10.1007/s00540-015-2105-X

[11] Baldo, B.A. and Fisher, M.M. (1993) Diagnosis of IgE-Dependent Anaphylaxis to Neuromuscular Blocking Drugs, Thiopentone and Opioids. Annales Françaises d'anesthèsie et de Rèanimation, 12, 173-181. http://dx.doi.org/10.1016/S0750-7658(05)81027-5

[12] Fisher, M.M. and Baldo, B.A. (2000) Immunoassays in the Diagnosis of Anaphylaxis to Neuromuscular Blocking Drugs: The Value of Morphine for the Detection of IgE Antibodies in Allergic Subjects. Anaesthesia and Intensive Care, 28, 167-170.

[13] Fisher, M. and Baldo, B.A. (1994) Anaphylaxis during Anaesthesia: Current Aspects of Diagnosis and Prevention. European Journal of Anaesthesiology, 11, 263-284.

[14] McDonnell, N.J., Pavy, T.J., Green, L.K. and Platt, P.R. (2011) Sugammadex in the Management of Rocuronium-Induced Anaphylaxis. British Journal of Anaesthesia, 106, 199-201. http://dx.doi.org/10.1093/bja/aeq366

[15] Raft, J., Belhadj-Tahar, N. and Meistelman, C. (2014) Slow Recovery after Sugammadex Bolus after Rocuronium-Induced Anaphylaxis. British Journal of Anaesthesia, 112, 1115-1116. http://dx.doi.org/10.1093/bja/aeu167 